J Clin Invest 67:319-327

28. Sauerheber RD, Kuhn CE, Hyslop PA 1984 Membrane structural/functional properties of adipocytes from normal and streptozotocin-diabetic rats. Diabetes $33: 258-264$

29. Scatchard G 1947 The attraction of proteins for small molecules and ions. Ann NY Acad Sci 51:660-672

30. Simmons MA, Jones MD, Battaglia FC, et al 1978 Insulin effect on fetal glucose utilization. Pediatr Res 12:90-92

31. Sinha MK, Miller JD, Ganguli S, Sperling MA 1983 Differential maturation of insulin sensitivity for glucose and amino acid metabolism in rat hepatocytes. Pediatr Res 335:17 (abstr)

32. Sperling MA 1978 In: Stave U (ed) Insulin and Glucagon in Perinatal Physiology. Plenum Press, New York, pp 813-829

33. Sperling MA, Delamater PV, Phelps D, Fiser RH, Oh W, Fisher DA 1974 Spontaneous and amino acid stimulated glucagon secretion in the immediate postnatal period. Relation to glucose and insulin. J Clin Invest 53:11591166

34. Sterman BM, Ganguli S, Devaskar S, Sperling MA 1983 Hypothyroidism and glucocorticoids modulate the development of hepatic insulin receptors. Pediatr Res 17:111-116

35. Thakur AK, Jaffe ML, Rodbard D 1980 Graphical analysis of ligand binding systems: evaluation by Monte Carlo Studies. Anal Biochem 107:279-295

36. Volpe JJ 1981 Hypoxic-ischemic encephalopathy: basic aspects and fetal assessment. In: Neurology of Newborn. WB Saunders Co, Philadelphia, M PCP XXII, pp 141-179.

37. Whitsett JA, Darovec-Beckerman C, Adams K, Pollinger J, Needleman H 1980 Thyroid dependent maturation of $\beta$-adrenergic receptor in the rat lung. Biochem Biophys Res Commun 97:913-917

38. Zamenof S, Burszytn H 1964 The determination of deoxyribonucleic acid and of cell number in brain. J Neurochem 11:505-510

\title{
Urinary Excretion of an Isomer of Bilirubin during Phototherapy
}

\author{
ISABELLA KNOX, JOHN F. ENNEVER, AND WILLIAM T. SPECK \\ Department of Pediatrics, Rainbow Babies and Childrens Hospital, Case Western Reserve University School of \\ Medicine, Cleveland, Ohio 44106
}

\begin{abstract}
Lumirubin, a water-soluble photoproduct of bilirubin formed in vivo during phototherapy, is excreted in the urine. In premature infants with little or no bilirubin conjugating activity, lumirubin is the principal yellow pigment found in the urine during phototherapy. The clearance rate of lumirubin in nine premature infants varied from 0.05 to $0.65 \mathrm{ml} / \mathrm{min}$ and increased with postconceptional age in parallel with increased creatinine clearance rate. The amount of lumirubin excreted per $24 \mathrm{~h}$ was estimated to be from 0.2 to $9.4 \mathrm{mg}$ with a mean of $3.2 \mathrm{mg}$. The urinary excretion of lumirubin is a significant pathway for pigment elimination during phototherapy. (Pediatr Res 19: 198-201, 1985)
\end{abstract}

Visible light phototherapy has been used to treat neonatal hyperbilirubinemia for more than two decades $(1,2)$. Although precise data are not available, it has been estimated that between 2 and $5 \%$ of all newborn infants are treated with phototherapy (3). Despite this widespread use over a number of years, the mechanism by which phototherapy lowers serum bilirubin in vivo is not known. The purpose of this study was to determine whether urinary excretion is an important pathway for the elimination of bilirubin photoproducts.

Bilirubin, a metabolic product of heme degradation (4), is a highly lipophilic molecule (5); prior to excretion, bilirubin is made more water soluble by conjugation to glucuronic acid (6).

Received July 12, 1984; accepted October 3, 1984.

Address correspondence and requests for reprints to John F. Ennever, Ph.D. M.D., Department of Pediatrics, Rainbow Babies and Childrens Hospital, 2101 Adelbert Road, Cleveland, $\mathrm{OH} 44106$.

Supported by the U.S. Public Health Service through Grants CA-23692 and Research Career Development Award 1K0-0043 to W.T.S
Because newborn infants are deficient in the enzyme(s) responsible for this conjugation reaction they frequently develop hyperbilirubinemia which is most often treated with phototherapy. During phototherapy, bilirubin undergoes two principal photochemical reactions, which yield products that are more polar than the native molecule (7-10). The relative importance of these two reactions to the therapeutic response seen with phototherapy depends on both the rates of formation and the rates of excretion of the photoproducts.

The two principal photoproducts are $4 Z, 15 E-b i l i r u b i n$, a configurational isomer of the native 4Z,15Z-bilirubin (9), and lumirubin, a structural isomer which contains a seven-member ring (10) (Fig. 1). The relative rates of the two reactions are known from in vitro studies (9-11) and appear to be similar in vivo (12). The faster reaction is the configurational isomerization which is freely reversible. The formation of lumirubin occurs more slowly (11) but is essentially irreversible. Typically during phototherapy, 2 to $6 \%$ of the total bilirubin is present as lumirubin whereas 15 to $20 \%$ is present as the configurational isomer (12). A third type of reaction, the photooxidation of bilirubin to mono and dipyrroles (13) occurs at a much lower rate than either isomerization reaction (11) and is not thought to be a quantitatively important pathway for bilirubin elimination.

The decline in serum bilirubin during phototherapy requires not only formation of these bilirubin isomers but also their elimination. The principal route of photoproduct elimination is thought to be through the bile. Onishi et al. (8) have reported finding a bilirubin photoproduct, which they called "unknown pigment," in the bile and urine of infants treated with phototherapy. We have used a high pressure liquid chromatographic method to quantitate the urinary excretion of bilirubin isomers in nine preterm infants. We have identified the photoproduct in the urine as the configurational isomer of bilirubin, lumirubin. We have determined the rate of urinary excretion of lumirubin 


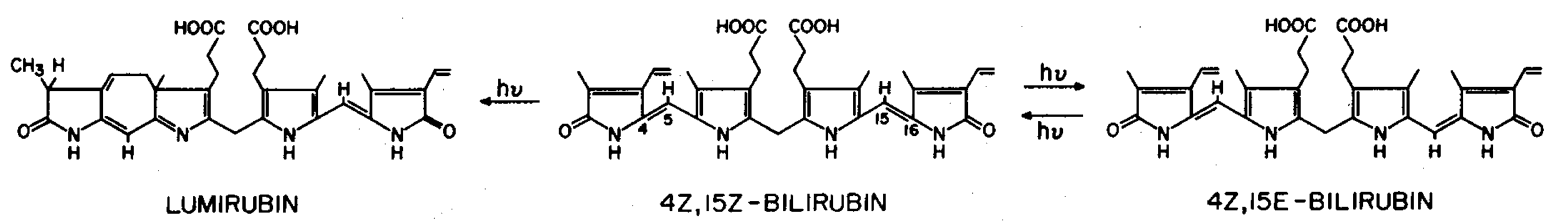

Fig. 1. Photochemical reactions of bilirubin. Native $4 Z, 15 Z$-bilirubin undergoes reversible configurational isomerization to $4 Z, 15 E-b i l i r u b i n$ $($ right $)$ and a slower essentially irreversible structural isomerization to lumirubin (left). Not shown in Figure 1 are slower photooxidative reactions.

Table 1. Characteristics of study population

\begin{tabular}{lcc}
\hline & Mean \pm SD & Range \\
\hline Birth wt (kg) & $1.8 \pm 0.8$ & $0.7-2.9$ \\
Gestational age (wk) & $31.9 \pm 4.0$ & $25-37$ \\
Age at study (days) & $3.9 \pm 1.6$ & $1-6$ \\
Hr under phototherapy at & $41 \pm 43$ & $5-120$ \\
$\quad$ time of study & & \\
\hline
\end{tabular}

and have found that it can account for only a fraction of the total bilirubin eliminated during phototherapy.

\section{METHODS}

Nine preterm infants were studied. The characteristics of the study population are given in Table 1 . During the collection period, the infants were treated with continuous phototherapy from a band of eight daylight fluorescent tubes housed in a standard phototherapy canopy. Urine was collected over a 24-h period in plastic neonatal urine collection bags, which were shielded from light during the collection by diapering the infant. The urine was kept in foil-wrapped containers until the collection was complete, then it was stored frozen until analyzed. During the 24-h collection period a blood sample was obtained under subdued lighting conditions and immediately placed in a foilwrapped tube. Creatinine concentration in urine and in serum was measured in the clinical chemistry laboratory at University Hospitals of Cleveland by standard techniques. All other work with serum and urine was done under photographic safelight.

Bilirubin photoproducts in serum and urine were analyzed by reversed-phase high pressure liquid chromatography on a $0.46 \times$ $25 \mathrm{~cm}$ Zorbax-ODS column (DuPont Instruments) equipped with a precolumn to trap the denatured protein from the serum samples. The eluant was $0.1 \mathrm{M}$ di- $n$-octylamine acetate in methanol, $\mathrm{pH} 7.7$ at a flow rate of $0.7 \mathrm{ml} / \mathrm{min}$ (9). Analyses were done on a Varian model 5060 liquid chromatograph equipped with a model UV-100 detector set at $465 \mathrm{~nm}$ or with a HewlettPackard model 1040A high speed scanning spectrophotometric detector with simultaneous monitoring at eight wavelengths including 450 and $465 \mathrm{~nm}$. The concentrations of bilirubin and bilirubin photoproducts were quantified from chromatographic peak areas by a Hewlett-Packard model $3390 \mathrm{~A}$ reporting integrator monitoring the absorbance at $465 \mathrm{~nm}$. The relative $465 \mathrm{~nm}$ extinction coefficients for 4Z,15Z-bilirubin:4Z,15E-bilirubin:lumirubin in $0.1 \mathrm{M}$ di- $n$-octylamine acetate in methanol are 1.0:0.92:0.42. These factors, which were used to correct the integrated peak areas on the chromatograms, were calculated from absorption spectra of the various isomers which were normalized at an isosbestic point. The identification of the photoproducts were based on chromatographic retention times and by absorption spectra obtained during chromatography by the high speed scanning spectrophotometric detector.

Clearances of creatinine and lumirubin were calculated by the standard formula:

$$
\text { Clearance }=\frac{\text { urine concentration } \times \text { urine volume }}{\text { plasma concentration }}
$$

The clearance results are expressed in $\mathrm{ml} / \mathrm{min}$. Bilirubin levels were measured at least twice per day by standard spectrophoto- metric methods in University Hospital's clinical chemistry laboratory. The serum concentration of lumirubin was estimated as the product of the mole-fraction of lumirubin in the serum from the high pressure liquid chromatography analysis and the average total bilirubin in the serum. The amount of lumirubin excreted per $24 \mathrm{~h}$ was calculated from the serum concentration and the clearance rate.

\section{RESULTS}

Figure 2 contains chromatograms of urine from a preterm infant obtained prior to the start of phototherapy (upper chromatogram) and after $8 \mathrm{~h}$ of phototherapy (lower chromatogram). Prior to phototherapy, the urine of preterm infants contains little material which absorbs $450 \mathrm{~nm}$ light (i.e., yellow pigment). After initiation of phototherapy, a yellow pigment appears in the urine. Identification of this photoproduct in the urine as the structural isomer, lumirubin, is based on the following: the urinary pigment has the same chromatographic retention time and the same absorption profile as lumirubin prepared by irradiation of bilirubin in vitro and purified as described by McDonagh et al. (10). In the absence of conjugates of bilirubin, lumirubin is the only yellow pigment excreted into the urine.

The clearance rates for lumirubin were measured in nine preterm infants and are plotted in Figure 3 as a function of postconceptional age (gestational plus postnatal age). For comparison, the creatinine clearance rates were simultaneously measured and the results (Fig. 3) were similar to those reported by Arant (14) for preterm infants. The clearance rate for lumirubin varied from 0.05 to $0.65 \mathrm{ml} / \mathrm{min}$ and increased with postconceptional age $(\mathrm{r}=0.70, p<0.05)$. The lumirubin clearance and creatinine clearance were highly correlated $(\mathrm{r}=0.96, p<0.01)$, with lumirubin clearance a consistent fraction $(8 \pm 4 \%)$ of creatinine clearance. The study sample was too small to distinguish the effect of postnatal age from the effect of gestational age on the clearance rates (14). The amount of lumirubin excreted in the urine per $24 \mathrm{~h}$ ranged from 0.2 to $9.4 \mathrm{mg}$ with a mean of $3.2 \mathrm{mg}$.

\section{DISCUSSIÓN}

Our results confirm the observation of Onishi et al. (8) that lumirubin is excreted in the urine of infants treated with phototherapy. Moreover, we have found that in premature infants with little or no bilirubin conjugating activity lumirubin is the principal yellow pigment excreted in the urine during phototherapy.

Lumirubin excretion in the urine must contribute to the therapeutic response to phototherapy; however, it does not account for the total pigment elimination during phototherapy. The total amount of bilirubin removed during phototherapy can only be estimated for the patients we studied. In the absence of a change in the total amount of bilirubin, elimination would equal production, which has been estimated to be $8.5 \mathrm{mg} / \mathrm{kg} /$ day in newborn infants (15). Using this estimate, bilirubin production in our study population would range from 6 to $25 \mathrm{mg} /$ day, which is greater than the urinary excretion rate of lumirubin we measured in these preterm infants $(0.2$ to $9.4 \mathrm{mg} / \mathrm{day})$.

Although lumirubin elimination in the urine is not responsible for the total excretion of pigment during phototherapy, it may 


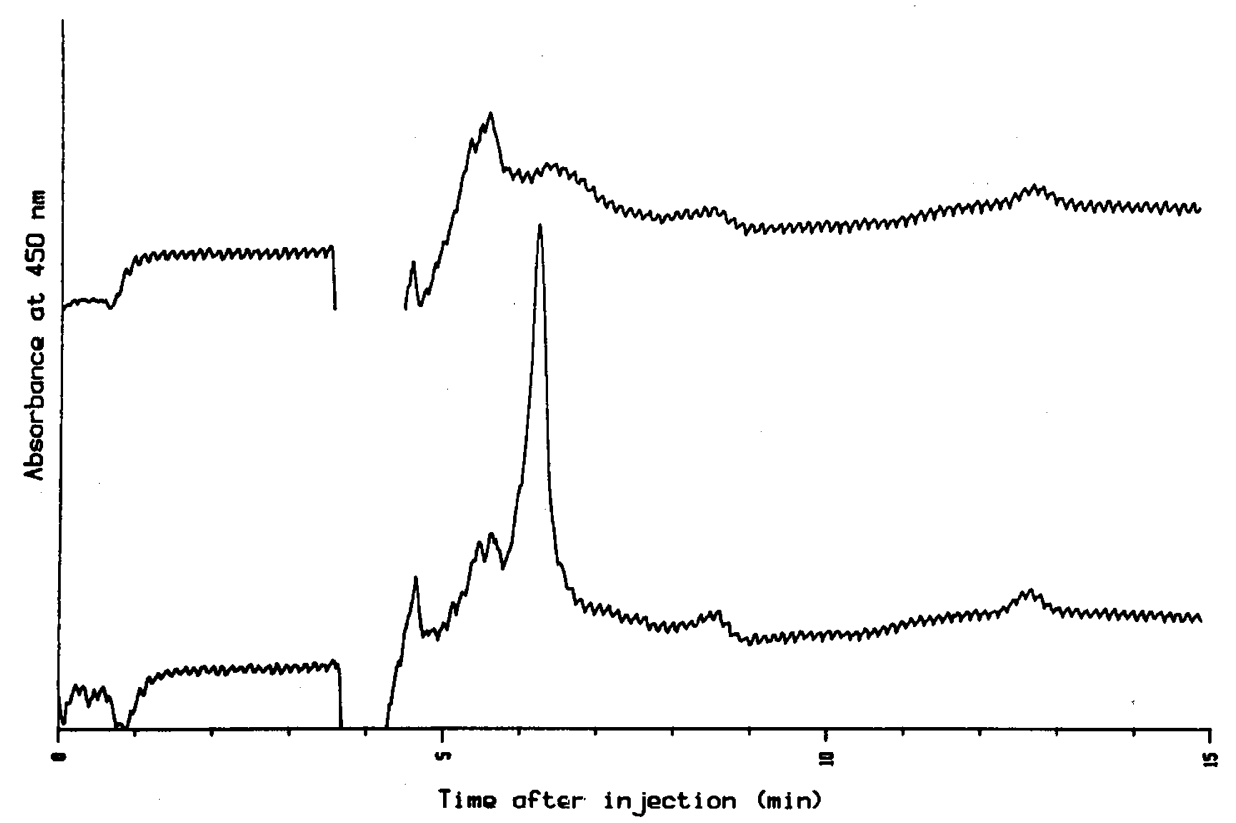

Fig. 2. High pressure liquid chromatography analysis of urine from one patient before (upper) and during (lower) phototherapy. Chromatography performed as described in "Methods." Negative deflection at 3.6 to $4.3 \mathrm{~min}$ is the injection artifact. Peak at retention time of approximately 6.2 min is lumirubin. Native bilirubin has retention time of $\sim 12.5 \mathrm{~min}$ in this high pressure liquid chromatography system.

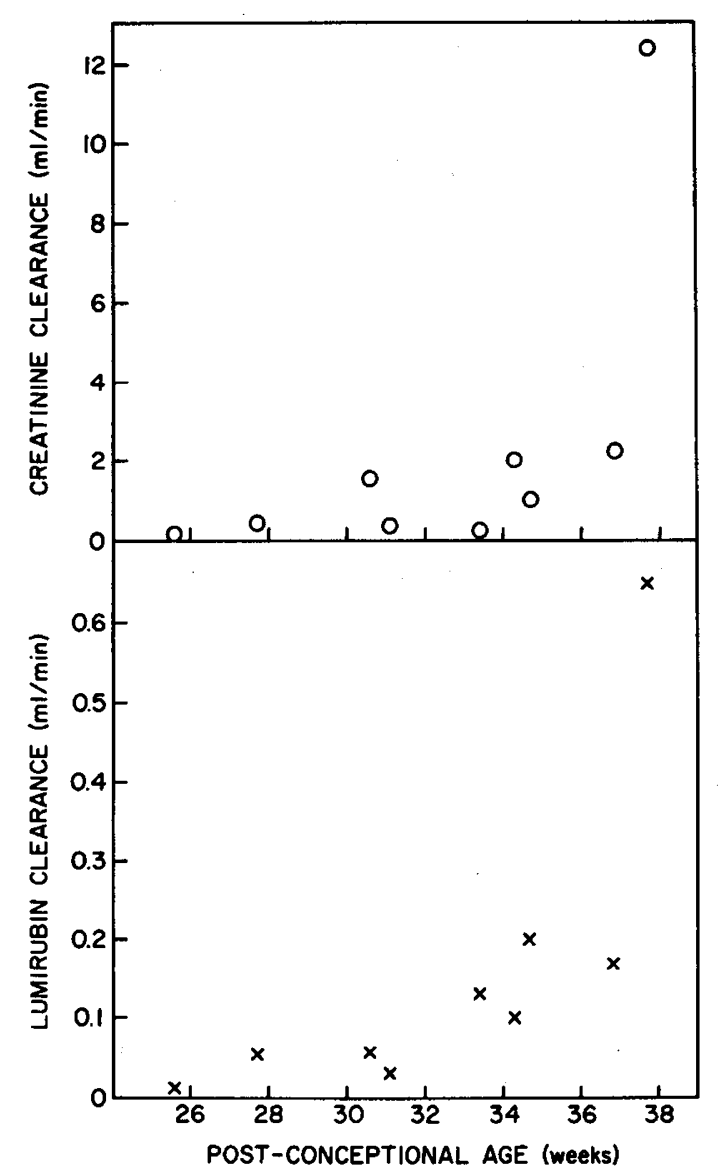

Fig. 3. Clearance rate for creatinine (upper) and lumirubin (lower) as a function of postconceptional (gestational plus postnatal) age. Both clearance rates were measured during the same 24-h period for each of the nine patients. Note the 20 -fold difference in the ordinal scales.

be a significant contributor. In 1970, Callahan et al. (16) reported on the excretion of bilirubin photoproducts in two infants (aged 5 and 7 months) with Crigler-Najjar syndrome. Following injection of a tracer dose of $\left[{ }^{14} \mathrm{C}\right]$ bilirubin, 18 to $28 \%$ of the excreted radioactivity were found in the urine ( 72 to $82 \%$ was in the stool). However, no diazo-positive material (intact bilirubin or bilirubin configurational isomers) was found in the urine. Although no chemical identification or structural analysis of the pigment derivatives was done by these investigators, the excreted $\left[{ }^{14} \mathrm{C}\right]$ labeled pigment most likely was lumirubin and photooxidation products, with lumirubin predominating because of its much faster rate of formation (11). If the mechanism of bilirubin elimination in premature infants is the same as in the older children with Crigler-Najjar syndrome studied by Callahan et al. (16), then as much as one-fourth of the pigment excreted during phototherapy may be the result of urinary clearance of lumirubin.

Lumirubin is formed from bilirubin by a photochemically irreversible reaction (Fig. 1). We have recently shown that the concentration of lumirubin in the serum of jaundiced infants can be increased by the use of higher intensity illumination during phototherapy (12), presumably from increased production. Furthermore, it may be possible to increase lumirubin production through use of selected wavelengths of light which favor formation of lumirubin over other photoproducts (17). Thus, increased intensity phototherapy with selected wavelengths of light may improve the efficacy of phototherapy and make phototherapy useful in clinical settings in which hepatic function is impaired.

\section{REFERENCES}

1. Cremer RJ, Perryman PW, Richards DH 1958 Influence of light on the hyperbilirubinaemia of infants. Lancet 1:1094-1097

2. Lucey J, Ferreiro M, Hewitt J 1968 Prevention of hyperbilirubinemia of prematurity. Pediatrics 41:1047-1054

3. Lewis HM, Campbell RHA, Hambleton G 1982 Use or abuse of phototherapy for physiological jaundice of newborn infants. Lancet 2:408-410

4. Ostrow JD, Jandl JH, Schmid R 1962 The formation of bilirubin from hemoglobin in vivo. J Clin Invest 41:1628-1637

5. McDonagh AF 1979 Bile pigments: bilatrienes and 5,15-biladienes. In: Dolphin D (ed) The Porphyrins, Vol VI. Academic Press, New York, pp 293-491

6. Schmid R 1957 The identification of 'direct-reacting' bilirubin as bilirubin diglucuronide. J Biol Chem 229:881-888

7. Stoll MS, Zenone EA, Ostrow JD, Zarembo JE 1979 Preparation and properties of bilirubin photoisomers. Biochem J 183:139-146

8. Onishi S, Isobe K, Itoh S, Kawade N, Sugiyama S 1980 Demonstration of a geometric isomer of bilirubin-IX $\alpha$ in the serum of a hyperbilirubinaemic newborn infant and the mechanism of jaundice phototherapy. Biochem $\mathbf{J}$ 190:533-536

9. McDonagh AF, Palma LA, Trull FR, Lightner DA 1982 Phototherapy for 
neonatal jaundice: configurational isomers of bilirubin. J Am Chem Soc 104:6865-6867

10. McDonagh AF, Palma LA, Lightner DA 1982 Phototherapy for neonatal jaundice: Stereospecific and regioselective photoisomerization of bilirubin bound to human serum albumin and NMR characterization of intramolecular cyclized photoproducts. J Am Chem Soc 104:6867-6869

11. Lamola AA, Flores J, Doleiden FH 1982 Quantum yield and equilibrium position of the configurational photoisomerization of bilirubin bound to human serum albumin. Photochem Photobiol 35:649-654

12. Costarino AT, Ennever JF, Baumgart S, Speck WT, Paul M, Polin RA 1985 Bilirubin photoisomerization in premature neonates under low and high dose phototherapy. Pediatrics (in press)

13. Lightner DA, Linnane WP'III, Ahlfors CE 1984 Bilirubin photooxidation products in the urine of jaundiced infants receiving phototherapy. Pediatr Res 18:696-700

14. Arant BS 1978 Developmental patterns of renal functional maturation compared in the human neonate. J Pediatr 92:705-712

15. Maisels MJ, Pathak A, Nelson NM, Nathan DG, Smith CA 1971 Endogenous production of carbon monoxide in normal and erythroblastotic newborn infants. J Clin Invest 50:1-8

16. Callahan EW, Thaler MM, Karon M, Bauer K, Schmid R 1970 Phototherapy of severe unconjugated hyperbilirubinemia: formation and removal of labeled bilirubin derivatives. Pediatrics 46:841-848

17. Ennever JF, Sobel M, McDonagh AF, Speck WT 1984 Phototherapy for neonatal jaundice: in vitro comparison of light sources. Pediatr Res 18:667670

\title{
The Preterm Rabbit: A Model for the Study of Acute and Chronic Effects of Premature Birth
}

\author{
ANTONIO V. LORENZO
}

Department of Neurosurgery, Children's Hospital, Boston, Massachusetts 02115

\begin{abstract}
The fetal rabbit delivered by caesarean section 0 to 5 days before term ( 32 days) can serve as a reliable animal model to study the short- and long-term consequences of premature birth. More than $80 \%$ of the fetal rabbits delivered at day 28 of gestation will survive $24 \mathrm{~h}$ if anesthetics are avoided during delivery and measures designed to meet the metabolic demands of extrauterine life are met. Sixty percent will survive up to and beyond the 7th day postpartum if the preterm pups are kept in a temperature and humidity controlled environment and are fed rabbit milk. Theoretical and practical advantages of this animal model are discussed. (Pediatr Res 19: 201205, 1985)
\end{abstract}

Laboratory animals delivered before term have been used successfully to study perinatal abnormalities associated with premature birth $(16,17,25)$. However, the inability to prolong survival beyond the first few hours of life has in most instances precluded the use of these animals in chronic studies of prematurity. The poor survival of the premature animal generally has been attributed to difficulties in establishing respiratory function immediately after birth and to the inability to provide effective nurturing postnatally $(4,21,23)$. A notable exception is the nonhuman primate whose ability to survive independently outside the uterus when delivered before term is well documented $(15,26)$. But the relative scarcity and the imposition of new restrictions on the acquisition and expense of monkeys has made the use of these animals in routine laboratory experiments prohibitive. The newborn rabbit delivered before term represents a practical and inexpensive alternative which in addition may be

Received May 29, 1984; accepted October 3, 1984.

Reprint requests to Antonio V. Lorenzo, Ph.D., Department of Neurosurgery, Children's Hospital, 300 Longwood Avenue, Boston, MA 02115.

This work was supported in part by the Vallely Family Fund and the Mental Retardation and Human Development Program of the Children's Hospital, Boston MA. a more appropriate model for the study of perinatal events such as intraventricular hemorrhage $(7,20,24)$.

\section{METHOD}

Female New Zealand White rabbits ranging in weight from 2.5 to $3.6 \mathrm{~kg}$ were purchased from commercial vendors and held in isolation for 5 to 7 days before being transferred to the breeding colony. The does were placed in individual cages $(40 \mathrm{H} \times 40 \mathrm{~W}$ $\times 52 \mathrm{D} \mathrm{cm}$ ) and given access to water, pelleted Rabbit Chow (Ralston-Purina Co., St. Louis, MO) and a salt lick ad libitum. Four to five adult bucks, housed individually in double sized cages $(40 \mathrm{H} \times 80 \mathrm{~W} \times 52 \mathrm{D})$, were used to service the does. The room was kept on a 12-h light-dark cycle, at $23 \pm 1.0^{\circ} \mathrm{C}$ and fresh air was circulated by exhaust fans.

On the day of mating (day 0 ) the doe was transferred to a bucks' cage and intromission was noted visually. To optimize the chance of fertilization the doe was mated sequentially with at least two bucks. Pregnancy could be verified as early as the 7th or 8th day following mating by palpating the uterine horns through the abdominal wall. The doe was kept on the same diet throughout pregnancy.

The litters were delivered by caesarean section 5 to 0 days before term (range 31 to 33 days). On the day of delivery the doe was placed in a restraining box and $50 \mathrm{ml}$ of air was injected rapidly into a marginal ear vein. Death ensued within 15 to 35 s. General anesthetics were avoided because the fetal rabbits either died in utero, failed to initiate spontaneous respiration, or succumbed shortly after birth when general anesthetics were used. Hysterotomy was usually accomplished within 4 to $8 \mathrm{~min}$; the time depending primarily on the size of the litter. After delivery the pharynx of each pup was aspirated and the pups were weighed and coded. To enhance survival newborn rabbits were injected intraperitoneally with $0.2 \mathrm{ml}$ of a $10.8 \mathrm{mM}$ glycerol or glucose solution immediately and again at 4 and at $8 \mathrm{~h}$ after birth. These injections were discontinued after the 1st yr of the study when it became apparent that glycerol administration was not required to enhance the survival of preterm animals fed 